\title{
A RICH with aerogel: a study of refractive index uniformity
}

\author{
M. Alemi, T. Bellunato, M. Calvi, C. Matteuzzi, M. Musy, D. L. Perego \\ Università degli Studi di Milano Bicocca and INFN, Piazza della Scienza 3, I-20126 Milano (Italy) \\ S. Easo \\ CCLRC Rutherford Appleton Laboratory, Chilton, Didcot, OX11 0QX Oxon (UK)
}

\begin{abstract}
The use of aerogel as a radiator in the RICH detectors of $\mathrm{LHCb}$ is a challenge due to the hot environment of the hadron collider LHC. Large size tiles of silica aerogel were recently produced with unprecedented optical quality for such dimensions. Results of laboratory measurements and beam tests are briefly reported. A description of a method to measure the uniformity of the index of refraction within the tile is given.
\end{abstract}

Index Terms - RICH, silica aerogel, optical properties, detector ageing, humidity tests.

\section{INTRODUCTION}

$\mathbf{T}$ HE LHCb experiment [1] is equipped with two Ring Imaging Cherenkov detectors using solid and gaseous radiators to provide a powerful particle identification tool [2], [3]. Silica aerogel covers the momentum range $2-10 \mathrm{GeV} / \mathrm{c}$ for $\pi / K$ separation.

Depending on the manufacturing procedure, the aerogel can be either hygroscopic or hydrophobic. For LHCb RICH, hygroscopic quality produced at the Boreskov Institute of Catalysis (Novosibirsk) has been chosen. A big effort in R\&D activities has been put during the last few years in order to obtain large size blocks with high transparency and good uniformity with respect to the refractive index variations within a tile. Large size (with dimensions $200 \times 200 \times 50 \mathrm{~mm}^{3}$ ) silica aerogel tiles with unprecedented optical quality are now available. In this paper tests on tiles with dimensions $100 \times 100 \times 40 \mathrm{~mm}^{3}$ are presented.

\section{Optical Properties of Aerogel}

Silica aerogel consists in a linked network of $\mathrm{SiO}_{2}$ nanocrystals, with a size distribution peaked at about $5 \mathrm{~nm}$, separated by empty volumes whose size range from a few up to $50 \mathrm{~nm}$. This structure defines the optical properties of the aerogel.

Even though aerogel inherits the transparency of silica quartz, light diffusion is usually the factor limiting the performance of this material as a Cherenkov radiator. The dominant contribution to the total diffusion probability comes from the Rayleigh scattering mechanism. The scattering probability for a photon of wavelength $\lambda$ is proportional to $\lambda^{-4}$.

The absorption cross section is, within a wide range, wavelength independent and can be neglected when considering

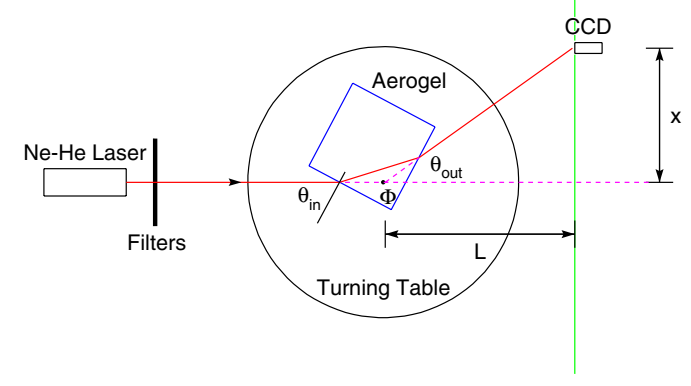

Fig. 1. Experimental setup used for the measurement of the refractive index.

samples a few centimetres thick. The transmittance $T$ of the aerogel can be parametrized by the Hunt formula [4]:

$$
T(\lambda)=\frac{I}{I_{0}}=A e^{-C \cdot t / \lambda^{4}}
$$

where $I_{0}$ and $I$ are the incident and the transmitted intensities of the light beam through the sample. $A$ is the surface scattering coefficient, $t$ is the thickness of the aerogel block, and $\lambda$ is the wavelength of the impinging light beam. $C$ is the clarity factor and it is used to specify the optical quality of a sample together with $A$. The transmittance $T$ is measured by means of a double beam spectrophotometer. A scan in the wavelength range between $200 \mathrm{~nm}$ and $800 \mathrm{~nm}$ is performed in steps of $1 \mathrm{~nm}$; subsequently the resulting curve is fitted to the Hunt formula (1) to extract $A$ and $C$. Typical values for the tiles are $C \simeq 0.0050 \mu \mathrm{m}^{4} / \mathrm{cm}$ and $A \simeq 0.95$.

In order to achieve a particle identification up to $10 \mathrm{GeV} / c$, the aerogel tiles developed for $\mathrm{LHCb}$ have a refractive index $n \simeq 1.03$. The refractive index $n$ of the aerogel is measured at the wavelengths $\lambda=543.5 \mathrm{~nm}$ and $\lambda=632.8 \mathrm{~nm}$ with an HeNe laser source. Measurements are performed using the prism method. Fig. 1 shows the block positioned in front of the laser beam on a turntable. The sample is rotated until the deflection angle $\theta_{\text {out }}$ reaches its minimum. In that condition the index of refraction is given by:

$$
n=\sin \left(\frac{\Phi+\theta_{\text {out }}}{2}\right) / \sin \left(\frac{\Phi}{2}\right)
$$




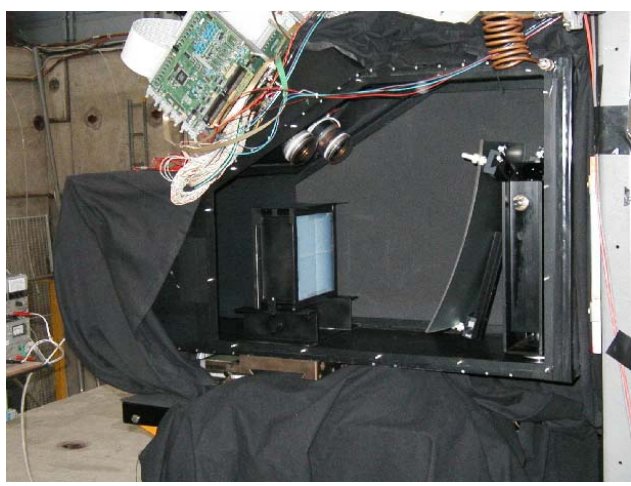

Fig. 2. A picture of the apparatus used for a test with Pixel HPDs.

where $\Phi$ is the angle between the two adjacent sides of the block $\left(\Phi \simeq 90^{\circ}\right)$; $\theta_{\text {out }}$, the deflection angle, is determined by measuring the displacement $x$ with a CCD camera located at $L=870 \mathrm{~mm}$ from the aerogel. The resolution achieved with this method is $\sigma_{n} \simeq 3 \times 10^{-4}$.

\section{Ageing EfFects in Aerogel}

Inside the LHCb detector, the aerogel radiator wall will be positioned at a radial distance of about $10 \mathrm{~cm} \mathrm{[2],} \mathrm{[3]} \mathrm{from} \mathrm{the}$ beam and about one metre downstream the interaction point. As a consequence, it will be exposed to a significant particle flux, up to $3.5 \times 10^{12}$ particles $/ \mathrm{cm}^{2} /$ year.

Possible ageing of aerogel due to intense irradiation has been investigated [5]. Aerogel tiles have been exposed to very intense $\gamma$ radiation from a ${ }^{60} \mathrm{Co}$ source and to proton and neutron high intensity beams. Transmittance has been monitored and the clarity factor $C$ has been studied as a function of the increasing dose of irradiation. The refractive index $n$ was also measured before and after irradiation.

No detectable degradation of the optical parameters has been observed for doses equivalent to a few times the LHCb lifetime in the case of $\gamma$ and proton irradiation. A small worsening of the clarity due to neutron irradiation has been observed. However, for a fluence corresponding to the LHCb lifetime, the clarity factor $C$ increases by about $5 \%$, and should not be a concern for the PID performance.

A test has been carried out to explore the behaviour of hygroscopic aerogel when exposed to humid air. A modification of its optical properties is expected. This test revealed that a prolonged exposure to humid air changes the optical properties of the aerogel, but they are completely restored by baking the exposed sample with a temperature of about $500^{\circ} \mathrm{C}$. In $\mathrm{LHCb}$, the aerogel will be mounted inside a gas tight box and it should never be exposed to humidity during running.

\section{Performance of Aerogel as Cherenkov RADIATOR}

Aerogel has been studied as Cherenkov radiator in several beam tests at CERN PS, using Hybrid PhotoDiodes (HPD) as photodetectors [6], [7]. In all these tests, the particle identifi- cation potential of an aerogel based RICH detector has been demonstrated using both pure $\pi^{-}$and mixed $\pi^{+} / p$ beams from the PS accelerator test beam line at CERN.

The Cherenkov emission angle $\theta_{C}$ is extracted from the photoelectron hit coordinates and the complete knowledge of the geometry of the setup using the retracking algorithm described in [8]. Beam test data indicate a resolution, per detected photoelectron, of $\sigma\left(\theta_{C}\right)=(4.8 \pm 0.3) \mathrm{mrad}$. Contributions to the resolution come from chromaticity, spherical aberrations, photodetector resolution, emission point spread and pixel dimensions [7].

Mixed $\pi^{+} / p$ beams of $6-10 \mathrm{GeV} / c$ give rise to two separate Cherenkov rings. The angular separation of the pion and proton rings $\Delta \theta_{C}=\theta_{C}(\pi)-\theta_{C}(p)$ is usually expressed in terms of the standard deviation $N_{\sigma}=\Delta \theta_{C} / \sigma_{C}(\pi)$. For positive pions and protons, a good separation power of the aerogel has been observed in the range studied, with $N_{\sigma}$ ranging from 17.1 to 7.8. For $\pi / K$ separation, these numbers would correspond to the range from 4.7 to 1.6 .

Recently, a test with the Pixel HPD [9], [10], which is the chosen photodetector for the RICH system of the LHCb experiment, has been carried out. The setup used is shown in Fig. 2, and the data are being analysed.

\section{STUdy OF REFRACTIVE INDEX UNIFORMITY}

The refractive index of the aerogel can be tuned during the production phase [11]. The refractive index $n$ and the density $\rho$ are related by:

$$
n(\lambda)=1+k(\lambda) \rho
$$

where $k$ is a wavelength-dependent coefficient. Typically, if the density is expressed in $\mathrm{g} / \mathrm{cm}^{3}, k=0.21$ at $\lambda=632 \mathrm{~nm}$. The knowledge of this coefficient is necessary for a fast and simple refractive index control during production.

Local inhomogeneities of the density lead to point to point variations of the refractive index within the monoliths. These variations contribute to the Cherenkov angle measurement accuracy. In order for the contribution to the total resolution on $\theta_{C}$ due to inhomogeneities not to be the dominant one, the maximum allowed refractive index variation $\sigma(n-1) /(n-1)$ is $1 \%$, corresponding to an uncertainty $\sigma\left(\theta_{C}\right) \simeq 1.17 \mathrm{mrad}$ for $n=1.03$.

A method [12] to study possible refractive index variations, shown in Fig. 3, uses a laser beam directed perpendicular to the aerogel surface in order to measure deviations due to the aerogel medium; the deviation angle $\delta$ is proportional to the refractive index gradients, $\mathrm{d} n / \mathrm{d} y=n \cdot \delta / t$, being $t$ the thickness of the block. The variation on $n$ along the $y$ direction can be easily calculated from the measurements of $\delta(y)$ scanning all over the aerogel surface:

$$
\Delta n(y)=\frac{n}{t} \cdot \int_{y_{0}}^{y} \delta(y) \mathrm{d} y
$$

Results from one tile are plotted in Fig. 4. A limitation of this method is that only one wavelength at a time can be monitored, and in any case a limited number of wavelengths can be used. 


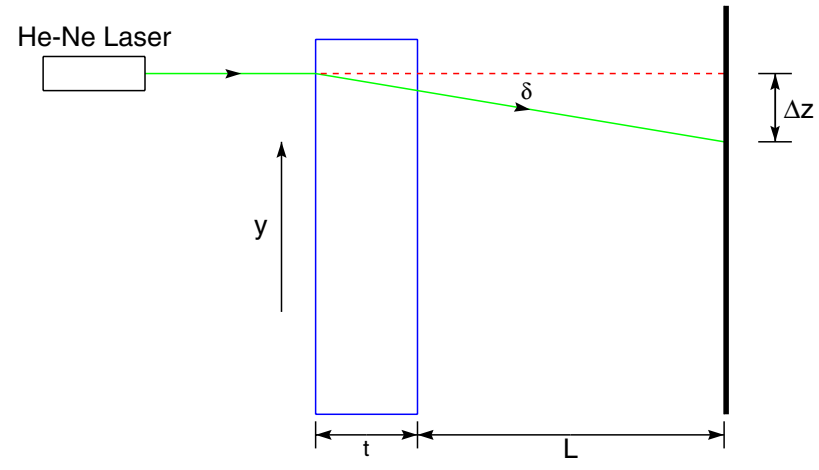

Fig. 3. Setup for the laser beam method used to study the refractive index uniformity.

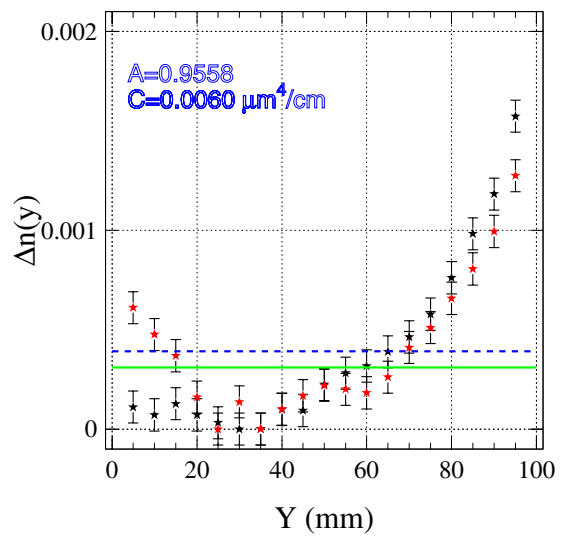

Fig. 4. Plot for the refractive index uniformity scan for one tile. The green solid line shows the maximum spread $\Delta n$ allowed, the blue dotted line shows the mean value of $\Delta n$ measured. The two set of points refer to two orthogonal scans performed on the same $100 \times 100 \times 41 \mathrm{~mm}^{3}$ tile.

An alternative method which makes use of a charged particle beam to test the uniformity of the refractive index has also been developed. This method exploits the Cherenkov effect itself, and it is therefore appropriate to check directly the influence of refractive index variations convoluted with the emission spectrum on the Cherenkov angle reconstruction performance.

A setup has been installed at the $500 \mathrm{MeV}$ electron beam of the DA $\Phi N E$ Beam Test Facility in Frascati [13], [14], [15]. The electron beam is used to scan the index of refraction varying the entrance point of the beam in the aerogel block. Black \& white photographic films have been used as photodetector. After each run of data taking, the exposed films have been processed by a professional photographic laboratory, and then used for the data analysis. The idea underlying these tests is that any local change in the refractive index reflects in a change of the distribution of the photons on the film.

Two configurations have been used, under the acronym "APACHE" for Aerogel Photographic Analysis by CHerenkov

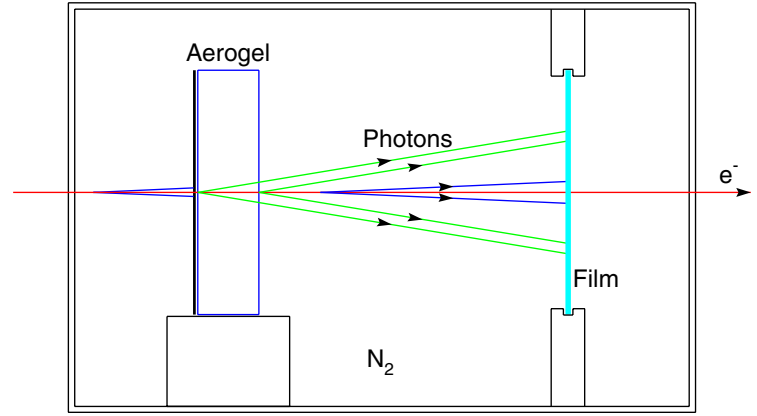

Fig. 5. The APACHE I setup used to measure the refractive index uniformity of the aerogel.

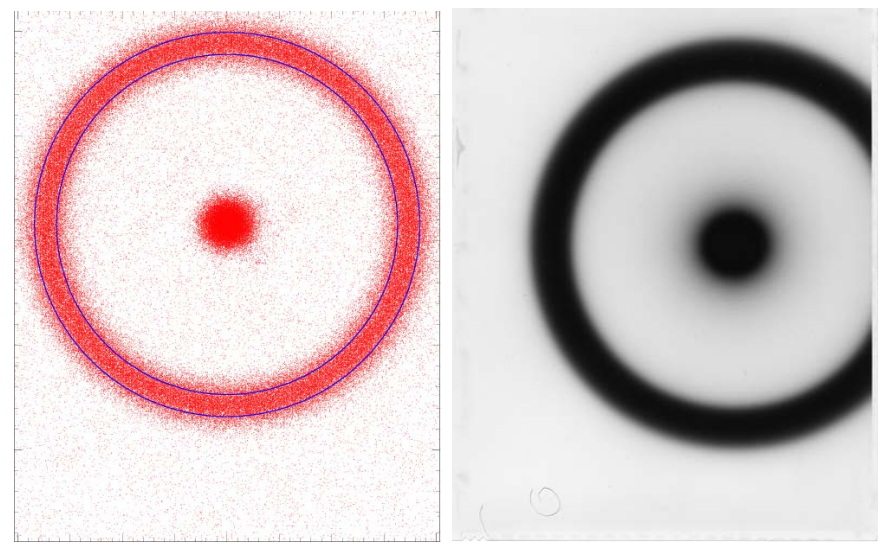

Fig. 6. A GEANT4 simulation (left) and data (right) of the photon distributions collected on a photographic film with the proximity focusing arrangement. Superimposed to simulation, two circles show the edges of the distribution foreseen in the absence of scattering, the width being due to the aerogel thickness only. For data film, part of the main ring has not been fully collected.

\section{Emission.}

\section{A. APACHE I}

The first configuration is a proximity focusing RICH detector. The setup, sketched in Fig. 5, consists of a dark room installed around a support for the aerogel tile to be tested, facing a 8 " $\times 10$ " black \& white photographic film ${ }^{1}$. The dark room volume is fluxed with nitrogen to avoid as much as possible a variation of the optical properties of the aerogel due to the absorption of humidity in the air [5]. A black paper screen is used to absorb the photons produced in nitrogen upstream the aerogel.

The Cherenkov photons produced in aerogel and nitrogen are directly collected on the photographic film, where their distribution forms a pair of concentric rings. A comparison between GEANT4 simulation [16] and data of the photon distributions is shown in Fig. 6 for the same tile tested with the laser method.

All the films are scanned and analysed. First, the centre of gravity of the nitrogen ring is calculated. Starting from this

\footnotetext{
${ }^{1}$ Kodak $\circledast$ Professional TRI-X 400 Film/400TX.
} 


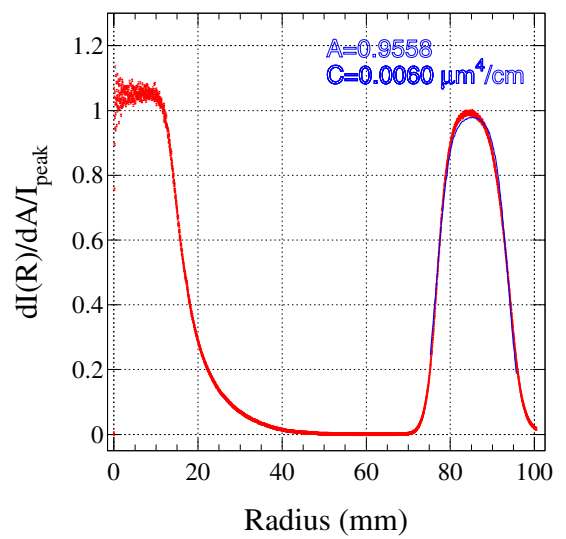

Fig. 7. The plot shows the radial, peak normalised light intensity distribution on a film exposed in a typical APACHE I run. In blue, the distribution is fitted with a gaussian modulated by the film response function.

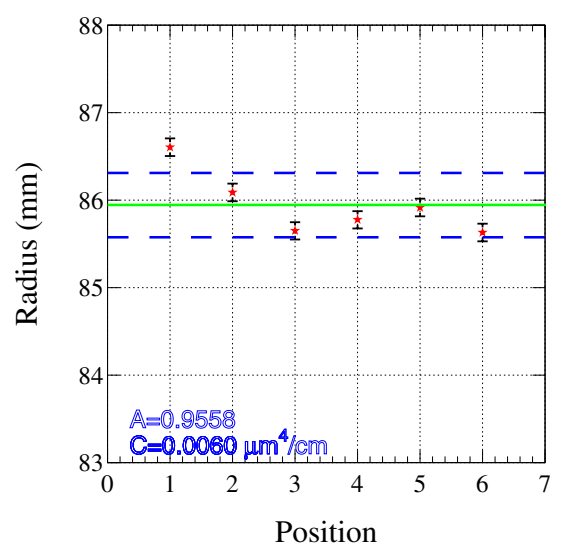

Fig. 8. Scatter plot of the radial positions of the peaks of all the APACHE I runs. The blue dashed lines bound the $1 \sigma$ region around the average value for $R$.

point, the radial distribution of the light intensity deposited on the film is built and fitted with a gaussian function convoluted with the appropriate threshold response of the film to multiple photon hits on the same grain. An example of a distribution resulting from such analysis is shown in Fig. 7.

Runs taken with the electron beam entering the aerogel in different positions allow to check for relative point to point variations of the refractive index within a tile. The simulation shows that, with this configuration, a point to point refractive index variation of $1 \%$ corresponds to $\Delta R=0.4 \mathrm{~mm}$, where $R$ is the peak position of the radial distribution. The resolution of APACHE I is $0.25 \mathrm{~mm}$, dominated by the thickness of the aerogel block. Results are summarized in Fig. 8, giving $\sigma(R)=$ $0.37 \mathrm{~mm}$.

In the same position, data were taken with and without a UV

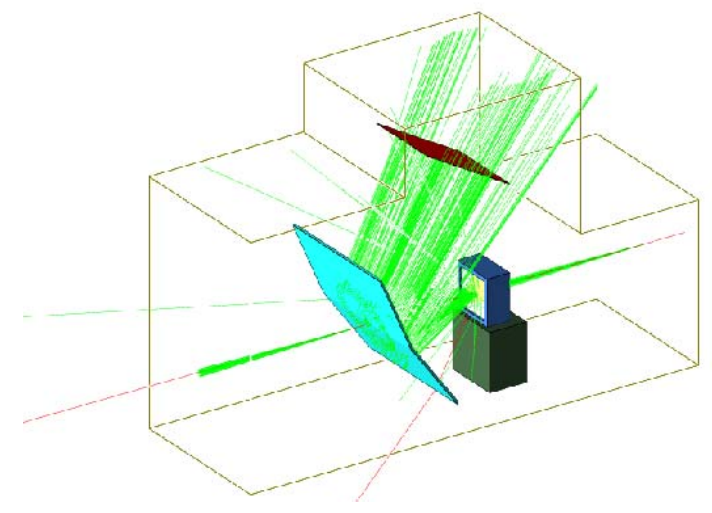

Fig. 9. Simulation of the experimental setup for the APACHE II.

filter of the same grade of the one which will be installed in the $\mathrm{LHCb}$ detector. The peak position is stable, but the distribution is significantly wider for runs without the filter, since the UV photons have a much higher probability of being scattered by the Rayleigh mechanism, as described in (1).

\section{B. APACHE II}

In order to improve the precision in the mean Cherenkov angle measurements and therefore to have a higher resolving power in terms of the refractive index inhomogeneities, the previous setup has been modified introducing a tilted spherical mirror to focus the Cherenkov photons onto the film, eliminating the radiator thickness effect which was the most limiting parameter. Fig. 9 shows the APACHE II configuration.

A different approach has been used for the analysis. An ideal mirror focuses all the photons with parallel trajectory to the same point in the focal plane, independent of the emission point and consequently of the aerogel thickness. Since the mirror is spherical and tilted with respect to the beam direction, spherical aberrations distort the focusing properties in a non trivial way. As a consequence, the Cherenkov rings on the detector plane are no longer perfectly circular, but they are distorted into roughly elliptical shapes. Fig. 10 shows a comparison between a full GEANT4 simulation and collected data. Rather than trying to fit such complex shapes, a significant simplification is obtained by calculating the Cherenkov emission angle $\theta_{C}$ that corresponds to a given combination of track direction and photon hit coordinate on the film. This calculation, referred to as reconstruction of the Cherenkov angle, can be solved analytically for a spherical focusing mirror [8].

The hit by hit retracking algorithm allows to build the distribution of the reconstructed Cherenkov angle, as shown in Fig. 11. A comparison of these distributions for each entering position give the shift of the mean angle $\theta_{C}$.

From the scan performed on the sample block, the results are shown in Fig. 12. A mean value $\theta_{C}=(245.96 \pm 1.14) \mathrm{mrad}$ has been obtained.

To study the uncertainties associated with this method, small changes in the setup geometry or beam conditions were sim- 


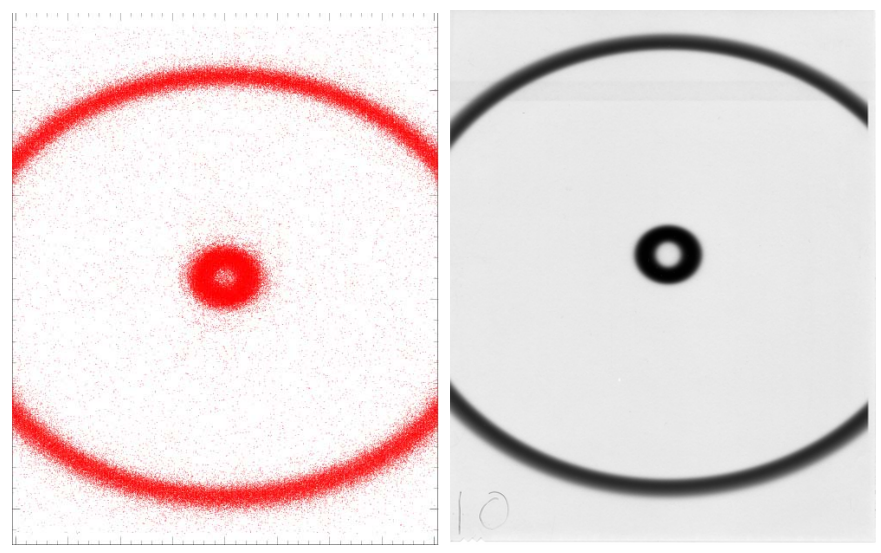

Fig. 10. A GEANT4 simulation (left) and data (right) of the photons collected onto the detection plane in APACHE with the focusing mirror (APACHE II).

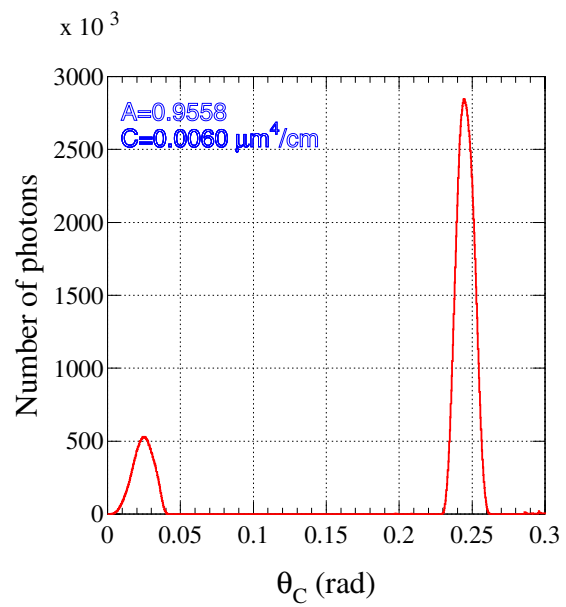

Fig. 11. The plot shows the distribution of the reconstructed angle $\theta_{C}$ in a typical APACHE II run.

ulated in different runs. The beam direction has been varied both as parallel displacement and as a tilt with respect to the nominal beam axis. The film position in space has been varied in order to check the effect of systematic shifts in the reconstruction parameters in the analysis. It has been found that small misalignments do not contribute substantially to the accuracy for measuring the relative variations of $n$.

To check the run conditions stability, a good indicator is the Cherenkov angle distribution for photons produced in the nitrogen volume between the aerogel and the mirror. The value of the angle for which the nitrogen ring has a maximum is independent of the aerogel, and it is expected to be constant from run to run, apart for tiny refractive index variations due to temperature and pressure changes. Any significant displacement of the nitrogen Cherenkov peak is then to be ascribed to uncontrolled variations of the run conditions. It has been verified by means of simulation that the resolution in the peak position is $0.3 \mathrm{mrad}$.

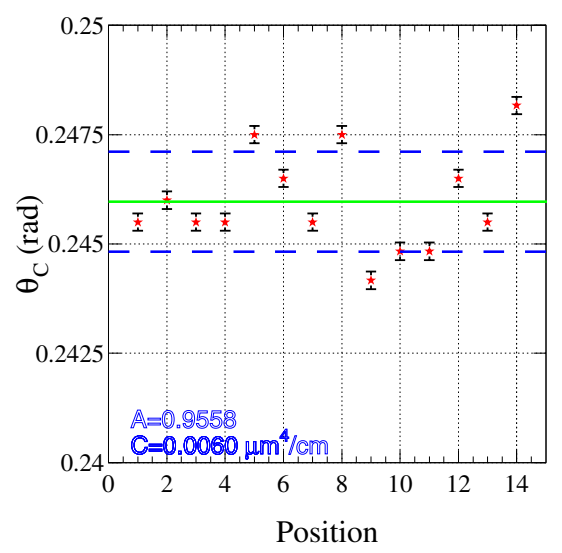

Fig. 12. Scatter plot of the mean reconstructed Cherenkov angle $\theta_{C}$ of all the APACHE II runs. The blue dashed lines bound the $1 \sigma$ region around the average value for $\theta_{C}$.

\section{CONCLUSION}

The aerogel project for the RICH detector of the $\mathrm{LHCb}$ experiment is well on track. The very promising results justify our confidence in getting large size aerogel blocks complying with the requirements of LHCb. All the optical parameters have been checked, and the final production has already started.

\section{ACKNOWLEDGMENT}

We acknowledge the financial support by INTAS contract No. 5579 for the aerogel R\&D program. The authors thank also F. Chignoli, R. Mazza, M. Melis, G. Mazzitelli, B. Buonomo and the staff of the DA $\Phi N E$ Beam Test Facility in Frascati for the help with the APACHE exeriment.

\section{REFERENCES}

[1] LHCb Coll., LHCb Technical Proposal, CERN/LHCC/98-4, 1998

[2] LHCb Coll., LHCb RICH TDR, CERN/LHCC/2000-0037, 2000.

[3] LHCb Coll., LHCb Reoptimized Detector Design and Performance, CERN/LHCC/2003-030, 2003.

[4] A. J. Hunt et al., Mat. Res. Soc. Symp. Proc. (1984) 275.

[5] T. Bellunato et al., Nucl. Instr. and Meth. A 527 (2004) 319.

[6] M. Alemi et al., IEEE Trans. Nucl. Sci. 48 (2001) 1265

[7] T. Bellunato et al., Nucl. Instr. and Meth. A 519 (2004) 493.

[8] R. Forty et al., Nucl. Instr. and Meth. A 384 (1996) 167.

[9] G. Aglieri Rinella, this conference proceedings.

[10] M. Patel, this conference proceedings.

[11] A. R. Buzykaev et al., Nucl. Instr. and Meth. A 379 (1996) 465.

[12] A. R. Buzykaev et al., Nucl. Instr. and Meth. A 433 (1999) 396.

[13] G. Mazzitelli et al., Nucl. Instr. and Meth. A 515 (2003) 524.

[14] B. Buonomo, this conference proceedings.

[15] http://www.Inf.infn.it/acceleratori/btf/

[16] S. Agostinelli et al., Nucl. Instr. and Meth. A 506 (2003) 250. 